\title{
THE JLAB 12 GEV ENERGY UPGRADE OF CEBAF FOR QCD AND HADRONIC PHYSICS*
}

\author{
Lawrence S. Cardman and Leigh Harwood ${ }^{1}$ (for the JLab $12 \mathrm{GeV}$ project team) \\ Thomas Jefferson National Accelerator Facility, Newport News, VA 23606 US.
}

\begin{abstract}
CEBAF at Jefferson Lab is a 5-pass, recirculating cw electron linac operating at $\sim 6 \mathrm{GeV}$ and devoted to basic research in nuclear physics. The $12 \mathrm{GeV}$ Upgrade is a major project, sponsored by the DOE Office of Nuclear Physics, that will expand its research capabilities substantially by doubling the maximum energy and adding major new experimental apparatus. We anticipate that the project will receive Critical Decision 2 approval this year and begin construction in 2008 .

The research program motivating the Upgrade includes: the study of hybrid mesons, which involve excited states of the glue, to explore the nature of quark confinement; dramatic improvements in our understanding of the QCD structure of the hadrons through the extension of our knowledge of their parton distribution functions to high $\mathrm{x}_{\text {Bjorken, where they are dominated by underlying valence }}$ quark structure, and a program of nucleon "tomography" via measurements of the Generalized Parton Distributions (GPDs), a broad program of experiments in the physics of nuclei that aims to understand the QCD basis for the nucleon-nucleon force and how nucleons and mesons arise as an approximation to the underlying quark-gluon structure; and precision tests of the Standard Model through parity violating deep inelastic and Møller scattering.

The Upgrade includes: doubling the accelerating voltages of the linacs by adding 10 new high-performance cryomodules; the requisite expansion of the $2 \mathrm{~K}$ cryogenics plant and rf power systems to support these cryomodules; upgrading the beam transport system from 6 to $12 \mathrm{GeV}$ through extensive re-use and/or modification of existing hardware; and the addition of one recirculation arc, a new experimental area, and the beamline to it; and the construction of major new experimental equipment for the GPD, high- $\mathrm{x}_{\mathrm{Bjorken}}$, and hybrid meson programs. The presentation will describe the science briefly and provide some details about the accelerator plans.
\end{abstract}

\section{OVERVIEW}

\section{Scientific Motivation}

In the two decades since the design parameters of CEBAF were defined our understanding of the behavior of strongly interacting matter has evolved significantly, and important new classes of experimental questions have

\footnotetext{
* This work was supported by the U.S. Department of Energy Contract Number DE-AC05-06OR23177 under which Jefferson Science Associates, LLC operates Jefferson Lab

${ }^{1}$ Contact L. Harwood (Hharwood@jlab.orgH) with anvauestions
}

been identified that can be addressed optimally by a CEBAF-type accelerator at higher energy. The original design of the facility, coupled with developments in superconducting RF technology, makes it feasible to triple the initial design value of CEBAF's beam energy to $12 \mathrm{GeV}$ in a cost-effective manner, providing a new research tool capable of addressing the science.

The science motivating the $12 \mathrm{GeV}$ Upgrade includes breakthrough programs that will be launched in four main areas (they are described in detail in [1]):

- The experimental study of gluonic excitations in order to understand the fundamentally new dynamics that underpins all of nuclear physics: the confinement of quarks. Theoretical conjectures, now strengthened by lattice QCD simulations, indicate that the most spectacular new prediction of QCD, quark confinement, occurs through the formation of a string-like "flux tube" between quarks. This conclusion (and proposed mechanisms of flux tube formation) can be tested by determining the spectrum of the gluonic excitations of mesons (referred to as "hybrid" mesons).

- The Fundamental Structure of the Nuclear Building Blocks. A vast improvement in our knowledge of the fundamental structure of the proton and neutron can be achieved. Existing deep inelastic scattering (DIS) cross sections will be extended for the first time to cover the critical region where their basic three-quark structure dominates, and measurements of new deep exclusive scattering cross sections will open the door to a comprehensive characterization of these wavefunctions using the framework of the Generalized Parton Distributions. These data will provide access to information on the correlations among the quarks. These studies will be complemented by detailed measurements of elastic and transition form factors, determining the dynamics underlying the quark-gluon structure through measurements of their high-momentum-transfer behavior and providing essential constraints on their description.

- The Physics of Nuclei. A diversified program of measurements that (taken together with the hadron studies outlined above) aims to provide a firm intellectual underpinning for all of nuclear physics by answering the question: "How does the phenomenological description of nuclei as nucleons interacting via an effective interaction parameterized using meson exchange arise from the underlying dynamics of quarks and gluons?" 


\section{$6 \mathrm{GeV} \Rightarrow 12 \mathrm{GeV}$}

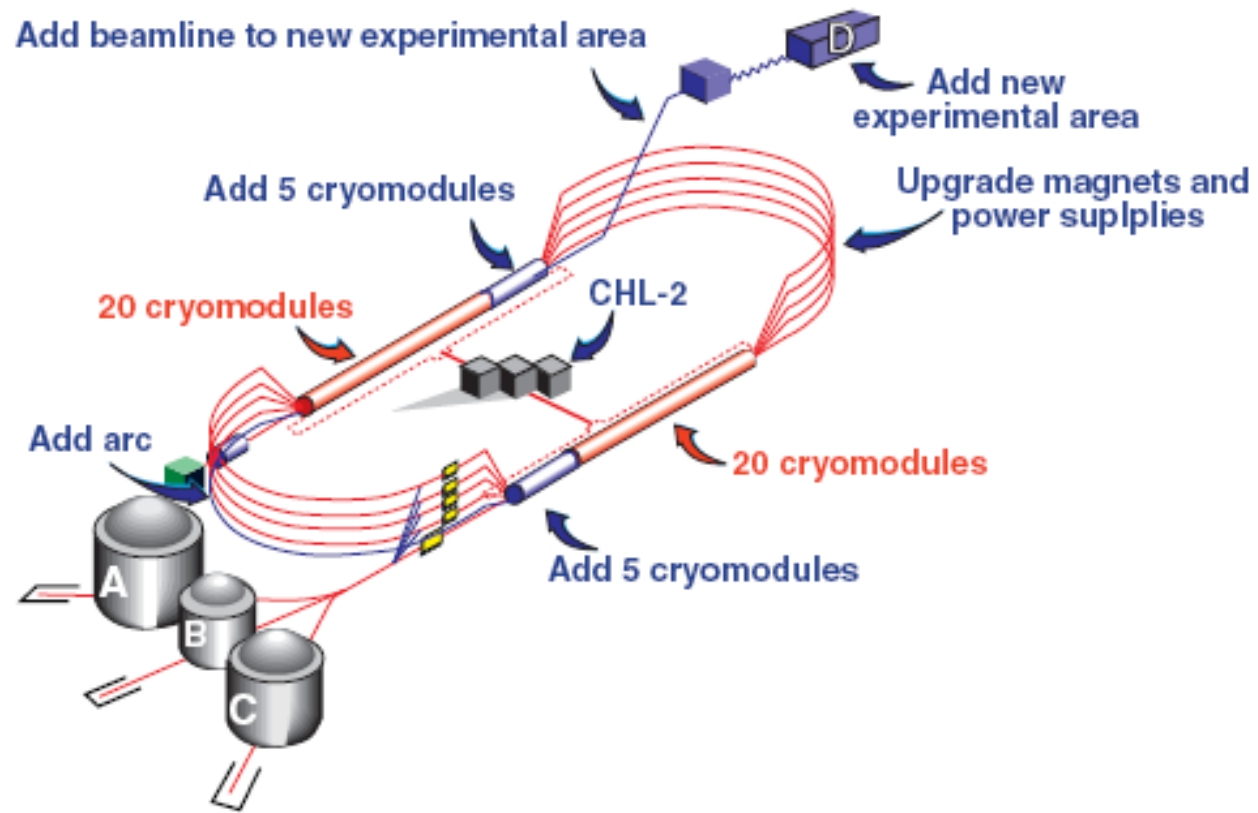

Figure 1: The layout of the CEBAF Accelerator with Upgrade enhancements identified

- Tests of the Standard Model of electro-weak interactions and the determination of fundamental parameters of this model. Precision, parity-violating electron scattering experiments made feasible by the Upgrade have the sensitivity to search for deviations from the Standard Model that could signal the presence of new physics. Other measurements of will lead to a significant improvement on our knowledge of chiral symmetry in QCD, in particular on the ratios of quark masses and on chiral anomalies.

\section{Base Requirements}

Careful studies identified a beam of $9 \mathrm{GeV}$ polarized photons as optimum for producing hybrid mesons and distinguishing them from the other meson excited states. Coherent bremsstrahlung, obtained by passing a high-quality, $\mathrm{cw}$ beam of $12 \mathrm{GeV}$ electrons through a crystal radiator, is the ideal source of these photons. Similar electron beam energies are ideal (or close to it) for the other research programs outlined above. The highquality $\sim 6 \mathrm{GeV} \mathrm{cw}$ electron beam capability of CEBAF and the infrastructure in place makes it an ideal platform upon which to base the new facility

The planning for the $12 \mathrm{GeV}$ Upgrade assumes that CEBAF has completed its evolution from the original $4 \mathrm{GeV}$ maximum beam energy to the present goal of $6 \mathrm{GeV}$. A program of refurbishment of the installed cryomodules is well underway, and will achieve this goal soon [2]. Thus, a net of $6 \mathrm{GV}$ of acceleration must be added. A new experimental area (for the hybrid meson program) will be added on the opposite end of the accelerator from three existing end stations, so the beam reaching it will transit one linac once more than beams for the existing end-stations (See Figure 1).

$12 \mathrm{GeV}$ can be achieved by increasing the linac capabilities or increasing the number of passes. An analysis determined that it would be more cost effective to increase the linac capabilities than to reconfigure (rebuild) the beam transport system for additional passes. Therefore, the present $0.6 \mathrm{GV}$ linacs will be upgraded to $1.1 \mathrm{GV}$. The hybrid meson studies need $<5 \mu \mathrm{A}$; the other programs need much larger beam currents. It was decided to retain the present beam power limit of $1 \mathrm{MW}$.

$12 \mathrm{GeV}$ can be achieved by increasing the linac capabilities or increasing the number of passes. An analysis determined that it would be more cost effective to increase the linac capabilities than to reconfigure (rebuild) the beam transport system for additional passes. Therefore, the present $0.6 \mathrm{GV}$ linacs will be upgraded to $1.1 \mathrm{GV}$. The hybrid meson studies need $<5 \mu \mathrm{A}$; the other programs need much larger beam currents. It was decided to retain the present beam power limit of $1 \mathrm{MW}$.

The present beam transport system supports 5-pass beam at $6 \mathrm{GeV}$ to the existing end-stations. It must be modified to deliver $11 \mathrm{GeV}$ beam to those halls and beam transport must be added to deliver the full $12 \mathrm{GeV}$ beam to the photon radiator target for the new end-station, including an additional recirculation arc.

\section{BEAM PHYSICS}

\section{Beam Breakup}

Beam breakup (BBU) driven by high-order modes (HOM's) in the cavities must always be addressed when 
using srf cavities even if the beam current in the cavities, $<1 \mathrm{~mA}$ in this case, is not exceptional on the scale of storage rings. As was done for $4 \mathrm{GeV}$ CEBAF, the code TDBBU [3] was used to evaluate the situation. An impedance specification of $Z<6 \times 10^{8} \Omega$ for the HOM's came out of the study. Initial tests of the new cavities indicate that there will be no problem with BBU for the Upgrade. A recent redesign of the cavity endgroups (discussed below) has resulted in a slight reduction in the damping but with a final result that is still within specification by over an order of magnitude.

\section{Emittance and Energy Spread}

Emittance growth from synchrotron radiation of the electrons in dispersive sections of the beam transport did not present a limitation to meeting the 1 nm-rad specification for CEBAF at $4 \mathrm{GeV}$. For the Upgrade, cost containment drove a desire to forego extensive modifications to the existing optics.

Table 1: Beam properties at 6 and $12 \mathrm{GeV}$

\begin{tabular}{|l|c|c|}
\cline { 2 - 3 } \multicolumn{1}{c|}{} & $6 \mathrm{GeV}$ & $12 \mathrm{GeV}$ \\
\hline Transverse emittance at & $\begin{array}{c}\varepsilon_{\mathrm{x}}=\varepsilon_{\mathrm{y}}= \\
1 \mathrm{~nm}-\mathrm{rad}\end{array}$ & $\begin{array}{c}\varepsilon_{\mathrm{x}}=7 \mathrm{~nm}-\mathrm{rad} \\
\varepsilon_{\mathrm{y}}=1 \mathrm{~nm}-\mathrm{rad}\end{array}$ \\
\hline $\begin{array}{l}\text { Transverse emittance at } \text { at } \\
\text { Hall D }\end{array}$ & NA & $\begin{array}{c}\varepsilon_{\mathrm{x}}=10 \mathrm{~nm}-\mathrm{rad} \\
\varepsilon_{\mathrm{y}}=2 \mathrm{~nm}-\mathrm{rad}\end{array}$ \\
\hline$\delta$ E/E at Halls A, B, \& C & $0.01 \%$ & $0.02 \%$ \\
\hline
\end{tabular}

Retaining those optics leads [4] to emittances at $11 \mathrm{GeV}$ in the existing end-stations of $\varepsilon_{\mathrm{x}}=7 \mathrm{~nm}-\mathrm{rad}$ and $\varepsilon_{\mathrm{y}}=1 \mathrm{~nm}$ rad. The projected energy spread at $12 \mathrm{GeV}$ is $0.02 \%$, as compared to $0.01 \%$ at $6 \mathrm{GeV}$. These values are consistent with the needs of the proposed research programs. Tracking calculations which included errors (misalignment, mispowering, field nonlinearities etc) have been carried out [5]. With all errors included in the model, the prediction is that the emittance and energy spread specifications will be met with some margin.

Halo

The hybrid meson experiment needs a very low beam halo to prevent unacceptable backgrounds. Particle-tracking calculations with multiple seeds for misalignment, missteering, field nonlinearities, and mispowering have shown that the halo requirement for commissioning and early years of operation will be met. Strategies for further reductions in the halo will be explored.

\section{ACCELERATION}

\section{Cryogenics}

The present $2 \mathrm{~K}$ helium plant is operating at its full capacity; any additional load, such as the new cryomodules, requires an expansion. JLab has a redundant $2 \mathrm{~K}$ coldbox identical to the primary $2 \mathrm{~K}$ coldbox. With a new $4 \mathrm{~K}$ coldbox, the potential addition of an $80 \mathrm{~K}$ exchanger, and utilization of the redundant $2 \mathrm{~K}$ coldbox, we will have a $2 \mathrm{~K}$ plant with maximum capacity of $\sim 5 \mathrm{~kW}$ at $2 \mathrm{~K}$. After allowance for system control headroom, there would be $\sim 300 \mathrm{~W}$ available at $2 \mathrm{~K}$ for each of the new cryomodules, (including 250W for fieldgenerated loads).

\section{$S R F$}

The additional $0.5 \mathrm{GV} /$ linac will be achieved by adding five new $100 \mathrm{MV}$ cryomodules to each linac. Each cryomodule will have eight 7-cell cavities. While only $17.5 \mathrm{MV} / \mathrm{m}$ is required for the cryomodule to reach $100 \mathrm{MV}$, the specification was set at $19.2 \mathrm{MV} / \mathrm{m}$ to deal with the likelihood (based on our operations experience with CEBAF) that some cavities might be off-line. The existing linacs could operate at the 5-pass equivalent of $5.6 \mathrm{GeV}$ if none of the margin is needed for off-nominal conditions in the cryomodules.

Typically, the performance specification for cavities is stated as a $\mathrm{Q}_{0}$ at a particular gradient. We have chosen to keep with the parameters determined by the overall system. For $12 \mathrm{GeV}$ we give a dynamic heat requirement rather than a $\mathrm{Q}_{0}$. After taking into account the load from the waveguides, the heat budget for dynamic load of each cavity is $29 \mathrm{~W}$. As presented at PAC05, prototypes have exceeded that requirement at $19.2 \mathrm{MV} / \mathrm{m}$. (Figure 2)

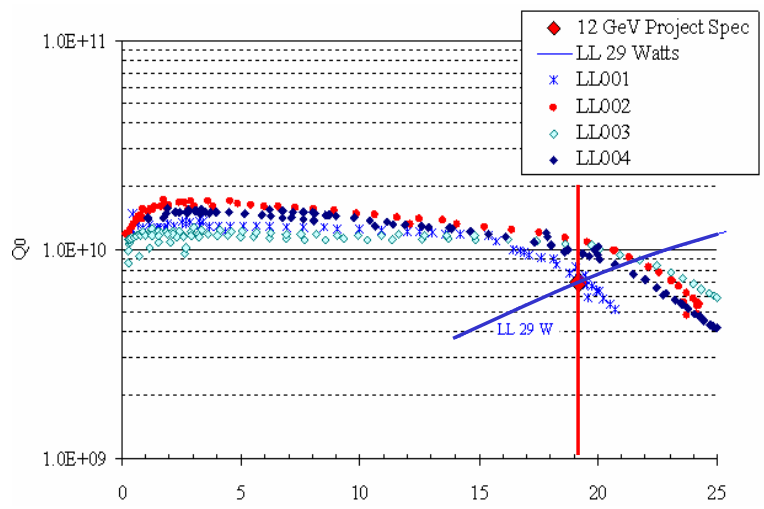

Figure 2: Cavity performance in early 2005 testing

It was also reported at PAC05 that a cryomodule (dubbed "Renascence") of the design intended for use in the $12 \mathrm{GeV}$ Upgrade was being built and would be tested in late 2005. Those tests occurred and had less-thanoptimal results; what appeared to be thermal management problems in the endgroups limited $\mathrm{cw}$ operation of the cavities to $<9 \mathrm{MV} / \mathrm{m}$. Temperature measurement hardware was added, some in situ exploratory design changes were made, and a set of investigatory measurements made. The finite-element thermal model was adjusted to agree with the data; the required adjustments provided identification of the problems. Three changes were made: 1) adjustment of the position and orientation of the higher-order mode (HOM) couplers, 2) elimination of the HOM couplers on the fundamental power coupler end (the damping is still $20 \times$ better than the specification), and 3 ) additional thermal strapping.

Two other changes have been made to the $12 \mathrm{GeV}$ Upgrade cryomodule design based on events in 2005:

- $\quad R F$ windows: First, we are returning to using both a primary and secondary rf window between the cavity and the external waveguide. This has been done because of unexplained failures in the ceramic rf windows used for 
the last cryomodule built for JLab's FEL. Those failures led to contamination of the cryomodule and seriously reduced performance. We decided that it would be too risky to put ten new cavities in CEBAF with the potential of such serious failures. The double-window configuration has been designed and included in the Project baseline.

- Tuner: A new, less-expensive "rock crusher" tuner concept was developed for Renascence. During the testing in 2005 we experienced some problems with mechanical robustness of the tuner. Subsequent to that, SNS had some problems with their cryogenic motors. An independent initiative had reworked the scissor-jack design for cost reduction and we returned to that design for improved reliability with little cost impact.

All of the design changes were incorporated into a $1 / 4-$ cryomodule that tested all components in a cryo-modulelike environment without incurring the cost and schedule delay of building a full cryomodule. This environment was critical for evaluating the design changes fully. The results from testing the $1 / 4$-cryomodule demonstrated that the design changes were fully successful [6]. Both of the cavities met the gradient and $\mathrm{Q}_{0}$ goals. Likewise, the tuners and $\mathrm{rf}$ windows met their goals. Consequently, all the redesigns have been accepted for the baseline $12 \mathrm{GeV}$ cryomodule design.

$R F$

Each cavity will be energized by its own klystron. The required saturated output power for the klystrons is based on the following criteria: 1) $\leq 450 \mu \mathrm{A}$ of beam transiting the cavity (limited by $1 \mathrm{MW}$ total beam power limit), 2) $\mathrm{Q}_{\text {ext }}$ is off-optimum by $\leq 30 \%, 3$ ) maximum detuning $\leq 25 \mathrm{~Hz}$ of which $4 \mathrm{~Hz}$ is $2 \times$ the tuner resolution and $21 \mathrm{~Hz}$ is $6 \mathrm{x}$ the $\sigma$ of the measured microphonics spectrum on existing cavities, 4) some cavities will be able to operate at $21 \mathrm{MV} / \mathrm{m}(10 \%$ above the mean of the population but within the range already demonstrated by testing of prototype cavities) and stay within the cryogenic budget, 5) losses in the waveguides, and 6) add $10 \%$ so that the klystron will still have gain. The result of the calculation was $12.8 \mathrm{~kW}$. $13 \mathrm{~kW}$ has been chosen as the design specification to add headroom. In 2006 a study was performed to determine the cost-effectiveness of powering mutilple cavities with a single klystron. It was found that cost reduction was possible but only at the expense of failing to meet the phase and amplitude control goals. This option was not adopted and $12 \mathrm{GeV}$ Upgrade will use one klystron per cavity.

A new rf control module is being developed using digital technology [7]. Important control issues that must be addressed are:

- Phase and amplitude control must meet the following specifications: 1) Amplitude noise $<0.01 \%$ and 2) Phase noise $<0.2^{\circ}$

- The system will have multi-valued resonance detuning curves resulting from the cavities' high fields and the accompanying high optimum external Q $\left(>2 \times 10^{7}\right.$ for $12 \mathrm{GeV}$ ), as illustrated in Figure 3.

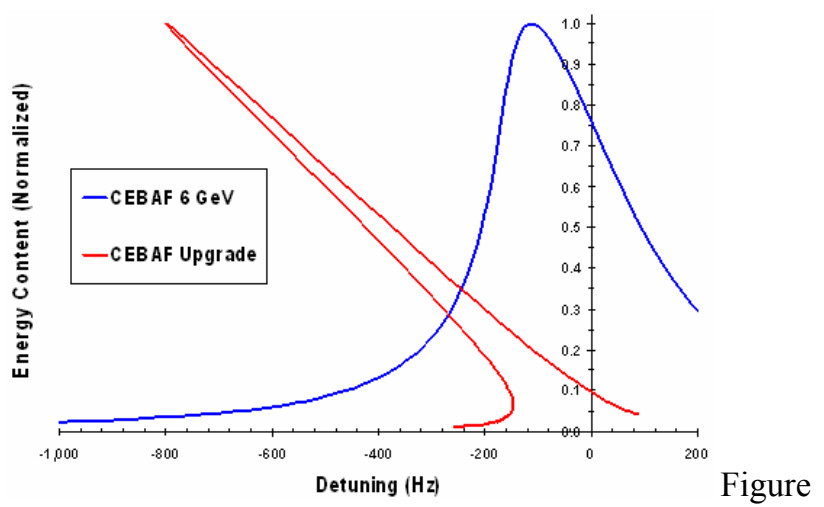

Figure 3: Detuning curves for 6 and $12 \mathrm{GeV}$

Successful testing of a technology demonstrator was done at JLab in November 2004 and January 2005. More recently successful tests were carried out of a unit developed at JLab which met the phase and amplitude control goals. Both of these units used generator-drive resonator (GDR) control algorithms. More recently still, a self-excited loop (SEL) digital algorithm has been developed [8] that shows great promise. This algorithm has the potential to eliminate the turn-on inconvenience caused by the double-valued detuning curve. While testing of cavities to date has only been at very low power, no show-stoppers were identified. We will continue to pursue this approach.

\section{BEAM TRANSPORT}

\section{Upgrading Existing Beamlines}

The existing beam transport system consists of $\sim 400$ dipoles $(\mathrm{B} \times \mathrm{L} \geq 0.2 \mathrm{~T}-\mathrm{m})$ and $\sim 700$ quads. Simply replacing their power supplies is not viable because saturation in the return iron would require excessively large power supplies and installation of a large refrigerated cooling-water system. Replacing the magnets is also cost prohibitive. Happily, another alternative was identified.

For the majority of the dipoles, i.e. those in the nine $180^{\circ}$ arcs and in the beamlines to the existing end-stations, the present " $\mathrm{C}$ " shape will be changed to " $\mathrm{H}$ " profiles by adding bolted-on iron plates. Modeling and prototyping have shown that the saturation is only a few percent with the additional iron, while it would exceed $50 \%$ if the iron were not added.

The dipoles in the spreaders and recombiners (S/R), i.e. the sections of the beam transport system which separate the co-linear beams after they exit the linacs or combine them before re-injection into the linacs, are so closely spaced that the bolt-on iron option is often not viable. A combination of three approaches will be used: 1) reshape the poles to reduce the flux, and thus the saturation, 2) add coil packs and accept the saturation, and 3 ) add iron, but in lesser amounts than in the $180^{\circ}$ arcs. We will replace 
magnets where no combination of these three options will work.

The quadrupole fields required for the Upgrade exceed the original design specifications of the present magnets for only $\sim 10 \%$ of the population. Tests were performed that showed satisfactory field quality if the units were pushed to the required field levels. The plan now is to replace the power supplies on that $10 \%$ and run them well in excess of their original design specification; the remainder will be left "as is".

The existing dipole strings are energized by 32 power supplies varying in size up to $750 \mathrm{~kW}$. Half will be reused for the Upgrade. The rest will be replaced by larger units that will use one or more common power units feeding current regulating units.

\section{New Beamlines}

The CEBAF accelerator's recirculating linac topology calls for specific ratios of each pass' energy. The acceleration in the injector directly affects those ratios. Thus, In order to match the increased linac voltages, the acceleration provided by the injector (nominally $0.11 \%$ of the 5-pass energy) much be increased. Two options were considered. The first was to replace one of the existing cryomodules with a new one and replace the associated $\mathrm{RF}$ zone; the second was to re-circulate the beam in the injector thereby doubling the acceleration. Recirculation has the lowest capital cost and was adopted as the baseline. Since re-circulation would require a longer commissioning period, that choice is being re-examined to insure that the total project cost (TPC) is minimized.

A new recirculation arc is needed to return the beam to the north linac for its final acceleration before going to Hall D. The optics for Arc 10 will be identical to that of Arcs 8 and 9. Both Arc 10 and the Hall D beamline must be built using new components; a total of forty-two $4 \mathrm{~m}$ "H"-style dipoles and 69 new quadrupoles are needed.

\section{Extraction}

A key to the effectiveness of CEBAF's research program is the ability to deliver beam to multiple halls simultaneously. This feature will be retained in the Upgrade. Either one or two of the bunch streams (the $1497 \mathrm{MHz}$ beam is made up of three independent but interleaved $499 \mathrm{MHz}$ bunch streams) can be extracted at any of the 5 passes. The extraction is accomplished use of a properly phased $499 \mathrm{MHz}$ horizontally deflecting copper cavity; the horizontal split is amplified by the downstream lattice and a septum. A second, stronger septum completes the separation into two independent beamlines, one going to the beam switchyard and the other entering the recirculation arcs.

The field integrals in all the devices must be doubled for the Upgrade. The additional rf field will be provided by increasing the number of cavities. The magnet integrals will be increased by adding magnets of existing designs in some locations and of new designs in other locations. The optics for all 5 passes will be identical.

\section{Diagnostics, Controls, and Safety Systems}

We presently anticipate no need to develop any new beam diagnostic instrumentation for this project. For the existing beamlines, no changes whatsoever are planned. New units of existing designs will be used for Arc 10 and for the beamline to Hall D. The control network will be extended using existing technology to accommodate the beamline to Hall D and Hall D itself.

All of the safety systems must be expanded because of the addition of Hall D to the complex. Specifically we must upgrade the Personnel Safety System (PSS), the Beam Envelop Limit System (BELS), and Machine Protection System (MPS). The MPS and BELS will be simple extension of the present system. A new engineering approach must be developed for the PSS in order to be in compliance with current regulations.

\section{PROJECT STATUS}

The $12 \mathrm{GeV}$ Upgrade project received approval of Mission Need, i.e. Critical Decision 0 (CD-0), from DOE in March 2004. Critical Decision 1 followed in February, 2006. It was identified this summer as the highest priority project in nuclear physics in the 2007 Long Range Plan of the Nuclear Sciences Advisory Committee. Later this summer the Project will undergo an External Independent Review by DOE; this review is the last review prior to approval of Critical Decision 2 (Approve Project Baseline). At this point, project completion, i.e. Critical Decision 4 (CD-4) is scheduled to be in FY15.

\section{SUMMARY}

An exciting new range of scientific inquiry into the quark nature of nuclear matter would be opened by the availability of a $12 \mathrm{GeV} \mathrm{cw}$ electron beam. JLab has developed a cost-effective plan that builds on its existing infrastructure to deliver the $12 \mathrm{GeV}$ beam. The project is progressing through the US Department of Energy's Critical Decision process with Critical Decision 2 scheduled for this year. The projected start of the science program is in FY14.

\section{REFERENCES}

[1] http://www.jlab.org/12GeV/

[2] .A. Drury, et al., WEPMS059, and J. P. Preble, et al WEPMS067 at this conference

[3] B. Yunn, JLab internal reports TN-01-028 and TN04-035.

[4] A. Freyburger, et al., at this conference.

[5] Y. Roblin, et al., THPMS065 at this conference.

[6] J. Preble, et al., WEPMS066 at this conference.

[7 P. Plawski, et al,, WEPMS065 at this conference.

[8] J. Hovater, et al., WEPMS060 at this conference. 